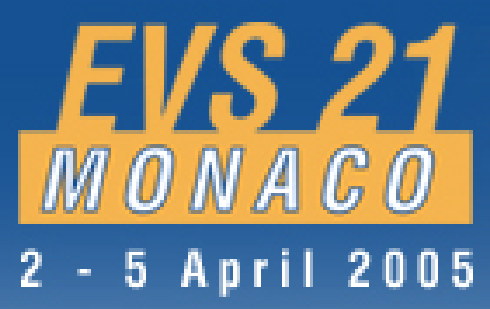

\title{
Energy Storage Fuel Cell Vehicle Analysis
}

\author{
Ahmad Pesaran \\ National Renewable Energy Laboratory \\ Golden, Colorado, USA
}




\section{Acknowledgments}

\section{Co-Authors}

Tony Markel (NREL)

Matthew Zolot (NREL)

Sam Sprik (NREL)

Harshad Tataria (GM/USABC)

Tien Duong (DOE)

Support and Guidance

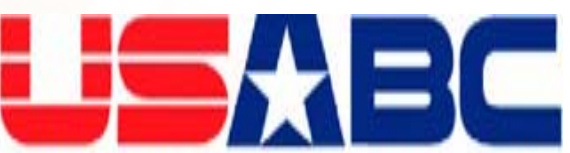

UNITED STATES ADVANCED BATTERY CONSORTIUM

FreedomCAR Energy Storage Technical Team

USABC Technical Advisory Committee

Ted Miller (Ford)

Cyrus Ashtiani (DaimlerChrysler)

Dave Howell (DOE) 


\section{Outline}

- Study Objectives

- Assumptions \& Requirements

- Analysis Approach

- Results

- Conclusions 


\section{Objective}

\section{Determine ESS Requirements for Fuel Cell Hybrid Vehicles for FreedomCAR Tech Teams}

\begin{tabular}{|c|c|c|}
\hline Freedom CAR Goals & & $\begin{array}{c}\text { Fuel Cell Hybrid } \\
\text { Battery }\end{array}$ \\
\hline Characteristics & Units & \\
\hline Pulse Discharge Power (x s) & $\mathrm{kW}$ & TBD \\
\hline Max Regen Pulse (y s) & $\mathrm{kW}$ & TBD \\
\hline Total Available Energy & $\mathrm{kWh}$ & TBD \\
\hline Round Trip Efficiency & $\%$ & $>90$ \\
\hline Cycle Life & Cyc. & TBD \\
\hline Cold-start at $-30^{\circ} \mathrm{C}$ (TBD kW for TBD $\mathrm{min}$.) & $\mathrm{kW}$ & TBD \\
\hline Calendar Life & Yrs & TBD \\
\hline MaxWeight & $\mathrm{kg}$ & TBD \\
\hline Max Volume & liters & TBD \\
\hline Production Price@100k units/yr & $\$$ & TBD \\
\hline Maximum Operating Voltage & $\mathrm{Vdc}$ & TBD \\
\hline Minim um Operating Voltage & $\mathrm{Vdc}$ & TBD \\
\hline Maximum Self Discharge & $\mathrm{Wh} / \mathrm{d}$ & 50 \\
\hline Operating Temperature & ${ }^{\circ} \mathrm{C}$ & -30 to +52 \\
\hline Survival Temperature & ${ }^{\circ} \mathrm{C}$ & -46 to +66 \\
\hline
\end{tabular}

Will these requirements will be different than ones for ICE-HEVs? 


\section{Vehicle Attributes \\ for Analysis}

\section{Forward-looking}

(What would be the vehicle characteristics when the fuel cell technology is ready and affordable?)

- Midsize

- Initial focus (popular)

- Needs smaller fuel cell, thus lower cost

- Extremely Lightweight

- For the same increase in fuel economy, it is more cost effective to take weight out of the vehicle rather than using a larger fuel cell

- Light weighting $\$ / \mathrm{kg}>>$ Larger fuel cell $\$ / \mathrm{kW}$

- Aerodynamic (relatively low drag coefficient) 


\section{Vehicle Assumptions Characteristics and Requirements}

\begin{tabular}{|c|c|c|}
\hline Characteristic & $\begin{array}{c}\text { Chevrolet Malibu } \\
\text { Model Year } 2004 \text { Mid-size car }\end{array}$ & $\begin{array}{c}\text { Final NREL Assumptions } \\
\text { for Mid-Size Car } \\
\text { (similar to Basic Malibu Sedan) }\end{array}$ \\
\hline $\begin{array}{c}\text { Dimensions }[\mathrm{mm}] \\
\text { (L x H x L-wheelbase) }\end{array}$ & $4,783 \times 1,460 \times 2,700$ & $4,749 \times 1,416 \times 2,656$ \\
\hline Curb Weight [kg] (calculated) & $1437 \mathrm{~kg}$ & TBD $\mathrm{kg}=1060 * 0.6+$ PT Mass \\
\hline Glider Mass [kg] & $1030(\mathrm{w} /$ driveline $)$ & $636=1060 * 0.6$ \\
\hline Weight Fraction (Front:Rear) [\%] & $63: 37$ & $50: 50$ \\
\hline Center-of-Gravity Height [m] & 0.5 - estimated & 0.45 \\
\hline Coefficient of drag & 0.30 & 0.25 \\
\hline Frontal Area $\left[\mathrm{m}^{2}\right]$ & 2.0 (calculated) & 2.0 \\
\hline Rolling Resistance Coefficient & $0.008-0.009$ & 0.0070 \\
\hline Range (composite City/highway) & $756 \mathrm{~km}$ & (320 miles) $500 \mathrm{~km}$ (minimum) \\
\hline Maximum Speed (FC only) & Better than $160 \mathrm{~km} / \mathrm{hr}$ & $(100 \mathrm{mph}) 160 \mathrm{~km} / \mathrm{h}$ \\
\hline$(0-60 \mathrm{mph}) 0-100 \mathrm{~km} / \mathrm{h}$ Acceleration & 11.0 seconds (estimate) & 11 seconds \\
\hline Gradeability (FC only) & Better than 6.5\% Grade@65 mph & $5.5 \%$ Grade @ 55 mph $(88$ km/h $)$ \\
\hline
\end{tabular}




\section{EI/S 21 \\ MO ONACO}

2 - 5 April 2005

The 21" Worldwide Battery, Hybrid and Fuel Cell Electric Vehicle Symposium \& Exhibition

- Mostly likely

$\checkmark 1$. Regenerative braking capture

$\checkmark 2$. Traction assist during acceleration (FC slow ramp rate)

$\checkmark 3$. Traction assist during high power transients (down sizing FC)

Probably

$\checkmark 4$. Traction assist during fuel cell "warm" startup from idle

$>$ Traction assist and power for ancillaries during fuel cell system "cold" startup \& shutdown

Probably not

- Sustained gradeability

- Electrical accessory loads (in steady state)

\section{Roles of}

Energy Storage System (ESS)

$\checkmark$ : assumed in this study

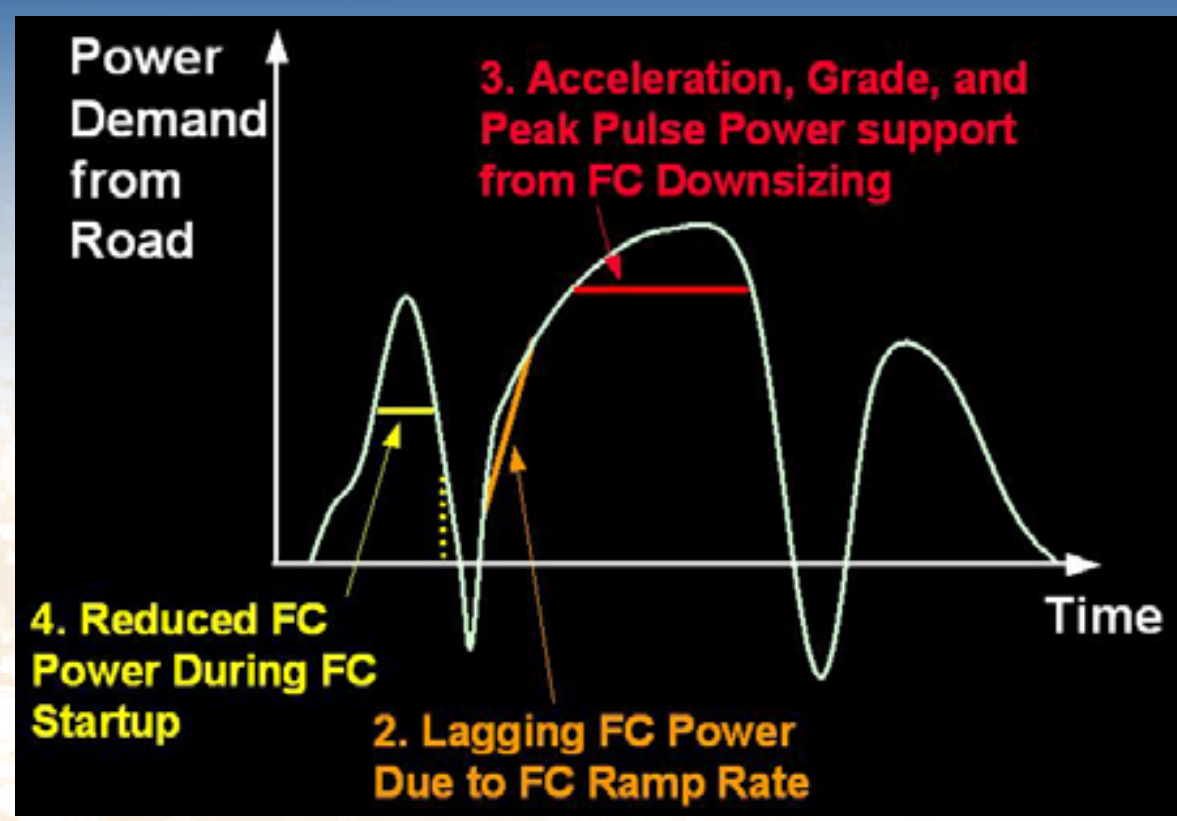

The combined FC and ESS hybridized system must meet performance target requirements of the vehicle

- Acceleration

- Top speed

- Grade sustainability 


\section{EIS 21 Other Assumptions}

MO ONAGO

2 - 5 April 2005

\section{FreedomCAR/DOE Targets}

\section{Fuel Cell}

\begin{tabular}{|l|c|c|}
\hline Assumption Description & Units & Value \\
\hline Fuel Type & -- & hydrogen \\
\hline Fuel Cell Peak Efficiency & $\%$ & 60 \\
\hline Fuel Cell Efficiency at 25\% Power & $\%$ & 60 \\
\hline Fuel Cell Efficiency at Rated Power & $\%$ & 50 \\
\hline Fuel Cell System Specific Power & $W / k g$ & 500 \\
\hline Fuel Cell System Power Density & W/L & 500 \\
\hline Fuel Cell System Cost & $\$ / k W$ & 32 \\
\hline $\begin{array}{l}\text { Fuel Cell System 10-90\% Power } \\
\text { Transient Response Capability }\end{array}$ & $\mathrm{s}$ & 1 \\
\hline $\begin{array}{l}\text { Time from Start to Full Power Output } \\
\text { Capability (20C) }\end{array}$ & $\mathrm{s}$ & 15 \\
\hline
\end{tabular}

- Assumed $700 \mathrm{~W}$ constant accessory loads

- Fuel cell is always on (i.e. no start/stop operation)

- Fuel cell net power is zero at vehicle stop/idle (gross power >0)

- At idle, hydrogen fuel consumption $0.3 \%$ of rated power consumption
Motor/Controller

\begin{tabular}{|l|c|c|}
\hline Assumption Description & Units & Value \\
\hline Specific Power (Motor and Controller) & $\mathrm{kW} / \mathrm{kg}$ & 0.75 \\
\hline Specific Cost (Motor and Controller) & $\$ / \mathrm{kW}$ & 12 \\
\hline Power Density (Motor and Controller) & $\mathrm{kW} / \mathrm{L}$ & 3.53 \\
\hline
\end{tabular}

Power Electronics

\begin{tabular}{|l|c|c|}
\hline Assumption Description & Units & Value \\
\hline Efficiency & $\%$ & 95 \\
\hline Specific Cost & $\$ / k W$ & 5 \\
\hline
\end{tabular}

Hydrogen Storage

\begin{tabular}{|l|c|c|}
\hline Assumption Description & Units & Value \\
\hline H2 Storage Energy Density & $\mathrm{kWh} / \mathrm{L}$ & 1.2 \\
\hline H2 Storage Specific Energy & $\mathrm{kWh} / \mathrm{kg}$ & 1.5 \\
\hline H2 Storage Cost & $\$ / \mathrm{kWh}$ & 4 \\
\hline
\end{tabular}




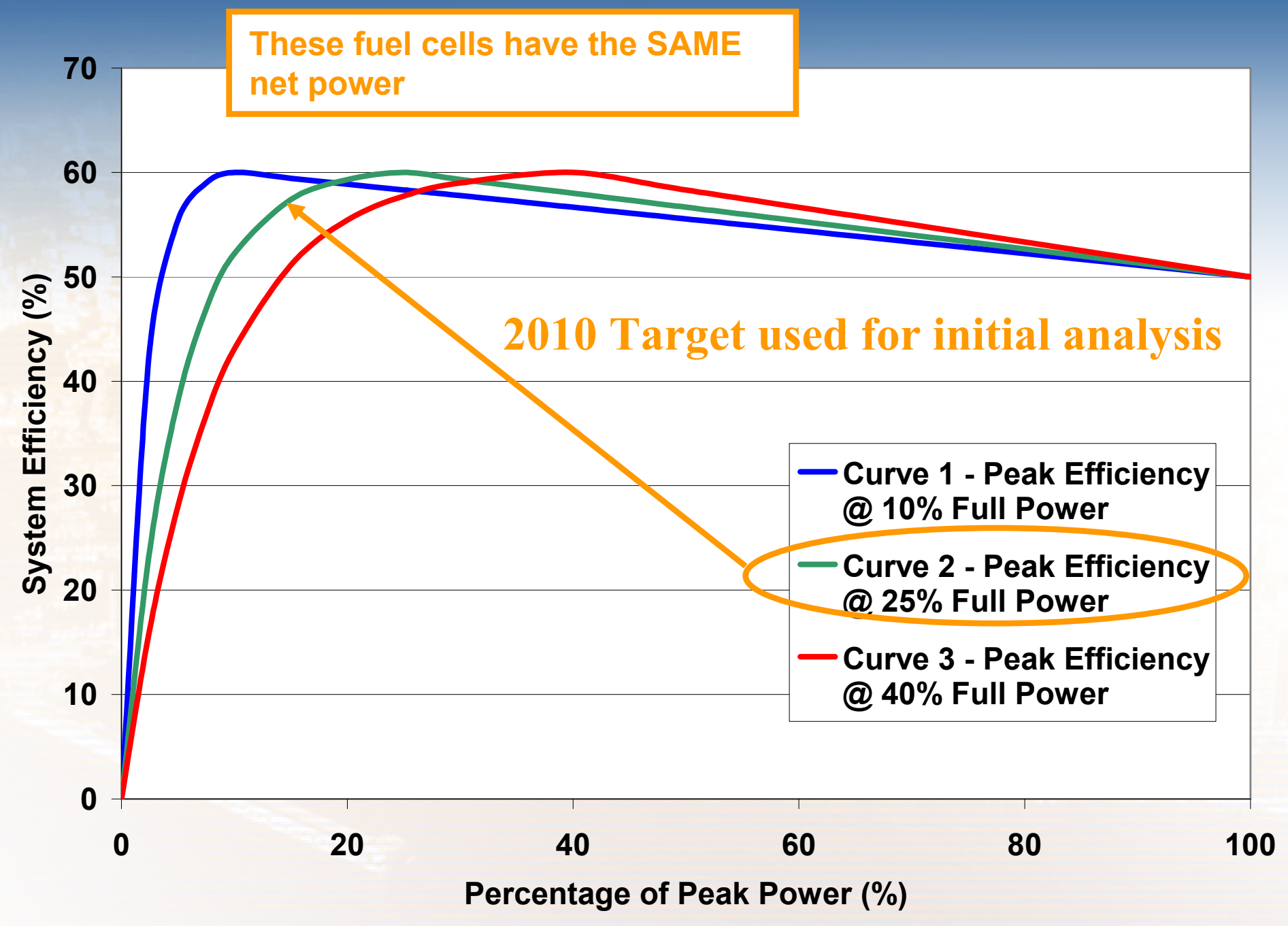




\section{Analysis Approach using ADVISOR ${ }^{\mathrm{TM}}$}

- Implemented an ESS control strategy (energy management) and regen capture strategy

- Simulated a range of FC/ESS configurations (Cases)

- Smallest fuel cell (Case1: sized for top speed)

- Cases 2-4 : incrementally increased fuel cell size; ESS sized to satisfy acceleration constraints

- Fuel cell only case (Case 5)

- Cases 5a-5c: ESS size increased to capture \% of peak regen pulse power

- Fuel cell plus ESS sized for max regen capture (Case 5a)

- Analyzed ESS power (kW) profile and Energy (Wh) history over several profiles

- Urban, Highway, US06 cycles, and European cycles

- Acceleration and gradeability tests 


\section{Energy Management Strategy/Assumption Used}

- Monitored changes in ESS and modified the fuel cell command to maintain ESS energy level

- Included

- Accounting of kinetic energy

- Opportunity charge and discharge functionality (only take action if it makes the overall system efficiency better)

- Monitored delta ESS energy to fuel-use ratio for correcting fuel economy

- Used multiple parameters to manage the strength of various elements of control

- A Design of Experiments was performed on each case to determine the best parameter settings

- Two Regenerative Braking Energy Capture

- A fraction of total possible

- Deceleration rate-based 
- Fractional split between driveline and friction brakes defined as a function of deceleration rate

\section{Regenerative Braking Strategy}

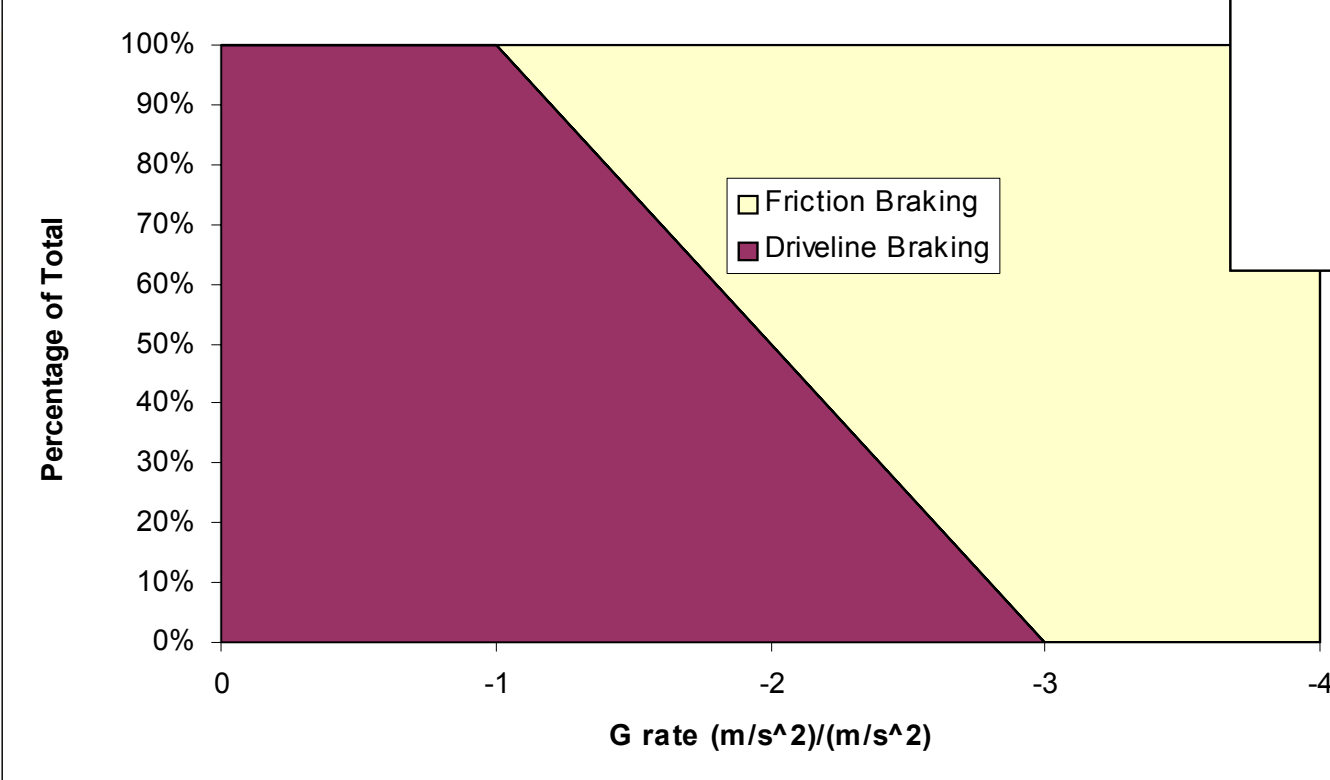

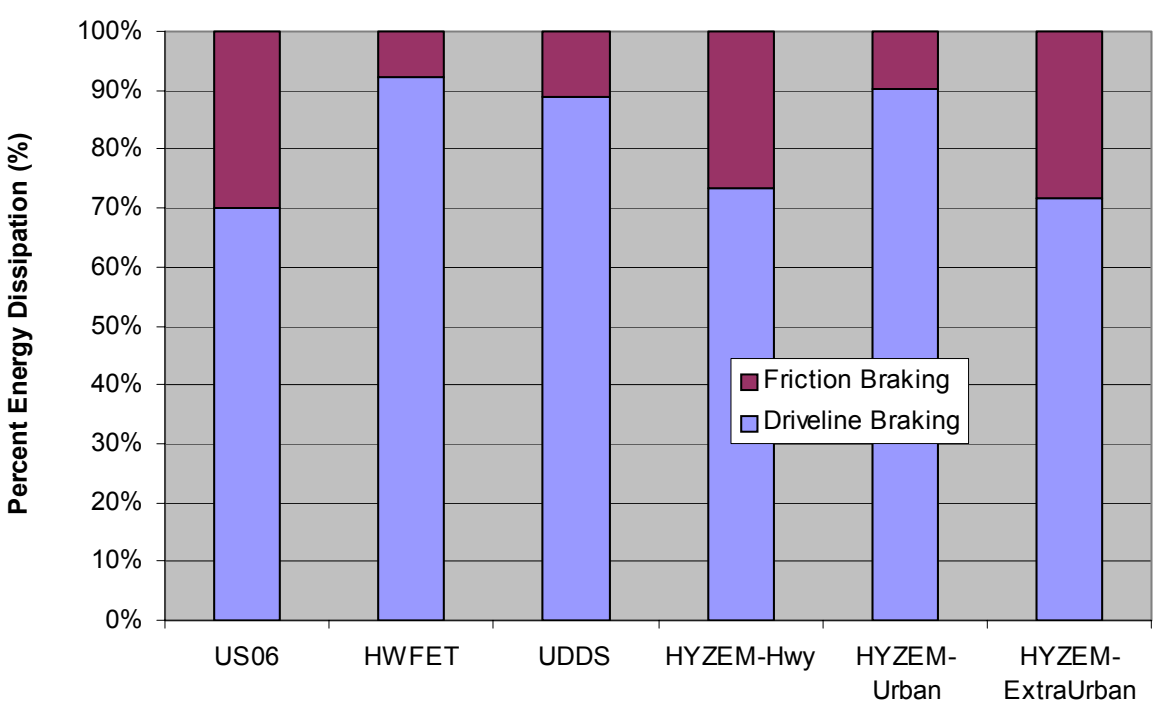

Driving Schedule
Given the current input assumptions (below $1 \mathrm{~g}$ all driveline braking, above $3 \mathrm{~g}$ all friction braking) the driveline would try to recapture between $70-90 \%$ of the available braking energy depending on the drive cycle. 
- Case 1-4 varies fuel cell size with "decelerationbased regen strategy"

- ESS sized to satisfy acceleration performance constraints

Case Xa-c with increasing regen power limits

Case Xf differs from Xa by the fuel cell characteristics

- Xa peak eff at 25\% power (DOE goal)

- Xf peak eff at $10 \%$ power

Power Requirements (FC-Only):

- Accel 0-60 mph: $75 \mathrm{~kW}$

- Top speed $100 \mathrm{mph}: 47 \mathrm{~kW}$

- Gradeability (55 mph @ 5.5\%): 34 kW

\begin{tabular}{|l|l|c|c|c|}
\hline \multicolumn{2}{|l|}{} & Fuel Cell & \multicolumn{2}{|c|}{ ESS } \\
\hline Name & Description & $(\mathbf{k W})$ & Regen (kW) & Discharge(KW) \\
\hline Case 1 & $\begin{array}{l}\text { FC sized for grade/top speed; decel regen } \\
\text { strategy; FC_FC50_P25 }\end{array}$ & 47000 & 34000 & 25000 \\
\hline Case 1a & Case 1 + 100\% regen & 47000 & 34000 & 25000 \\
\hline Case 1b & Case 1 + 75\% regen & 47000 & 25500 & 25000 \\
\hline Case 1c & Case 1 + 50\% regen & 47000 & 17000 & 25000 \\
\hline Case 1f & Case 1a + FC_FC50_P10 & 47000 & 34000 & 25000 \\
\hline Case 2 & $\begin{array}{l}\text { Fuel cell - sized to 25\% point; decel regen } \\
\text { strategy; FC_FC50_P25 }\end{array}$ & 54250 & 34000 & 18000 \\
\hline Case 2a & Case 2 + 100\% regen & 54250 & 34000 & 18000 \\
\hline Case 2b & Case 2 + 75\% regen & 54250 & 25500 & 18000 \\
\hline Case 2c & Case 2 + 50\% regen & 54250 & 17000 & 18000 \\
\hline Case 2f & Case 2a + FC_FC50_P10 & 54250 & 34000 & 18000 \\
\hline Case 3 & $\begin{array}{l}\text { Fuel cell - sized to 50\% point; decel regen } \\
\text { strategy; FC_FC50_P25 }\end{array}$ & 61500 & 34000 & 12500 \\
\hline Case 3a & Case 3 + 100\% regen & 61500 & 34000 & 12500 \\
\hline Case 3b & Case 3 + 75\% regen & 61500 & 25500 & 12500 \\
\hline Case 3c & Case 3 + 50\% regen & 61500 & 17000 & 12500 \\
\hline Case 3f & Case 3a + FC_FC50_P10 & 61500 & 34000 & 12500 \\
\hline Case 4 & $\begin{array}{l}\text { Fuel cell - sized to 75\% point; decel regen } \\
\text { strategy; FC_FC50_P25 }\end{array}$ & 69000 & 34000 & 7500 \\
\hline Case 4a & Case 4 + 100\% regen & 69000 & 34000 & 7500 \\
\hline Case 4b & Case 4 + 75\% regen & 69000 & 25500 & 7500 \\
\hline Case 4c & Case 4 + 50\% regen & 69000 & 17000 & 7500 \\
\hline Case 4f & Case 4a + FC_FC50_P10 & 69000 & 34000 & 7500 \\
\hline Case 5 & Fuel cell only - no ess; FC_FC50_P25 & 75000 & 0 & 0 \\
\hline Case 5a & Fuel cell only plus 100\% ess & 75000 & 36000 & 36000 \\
\hline Case 5b & Fuel cell only plus 75\% ess & 75000 & 27000 & 27000 \\
\hline Case 5c & Fuel cell only plus 50\% ess & 75000 & 18000 & 18000 \\
\hline Case 5f & Case 5 + FC_FC50_P10 & 75000 & 0 & 0 \\
\hline & & & & \\
\hline
\end{tabular}



120000

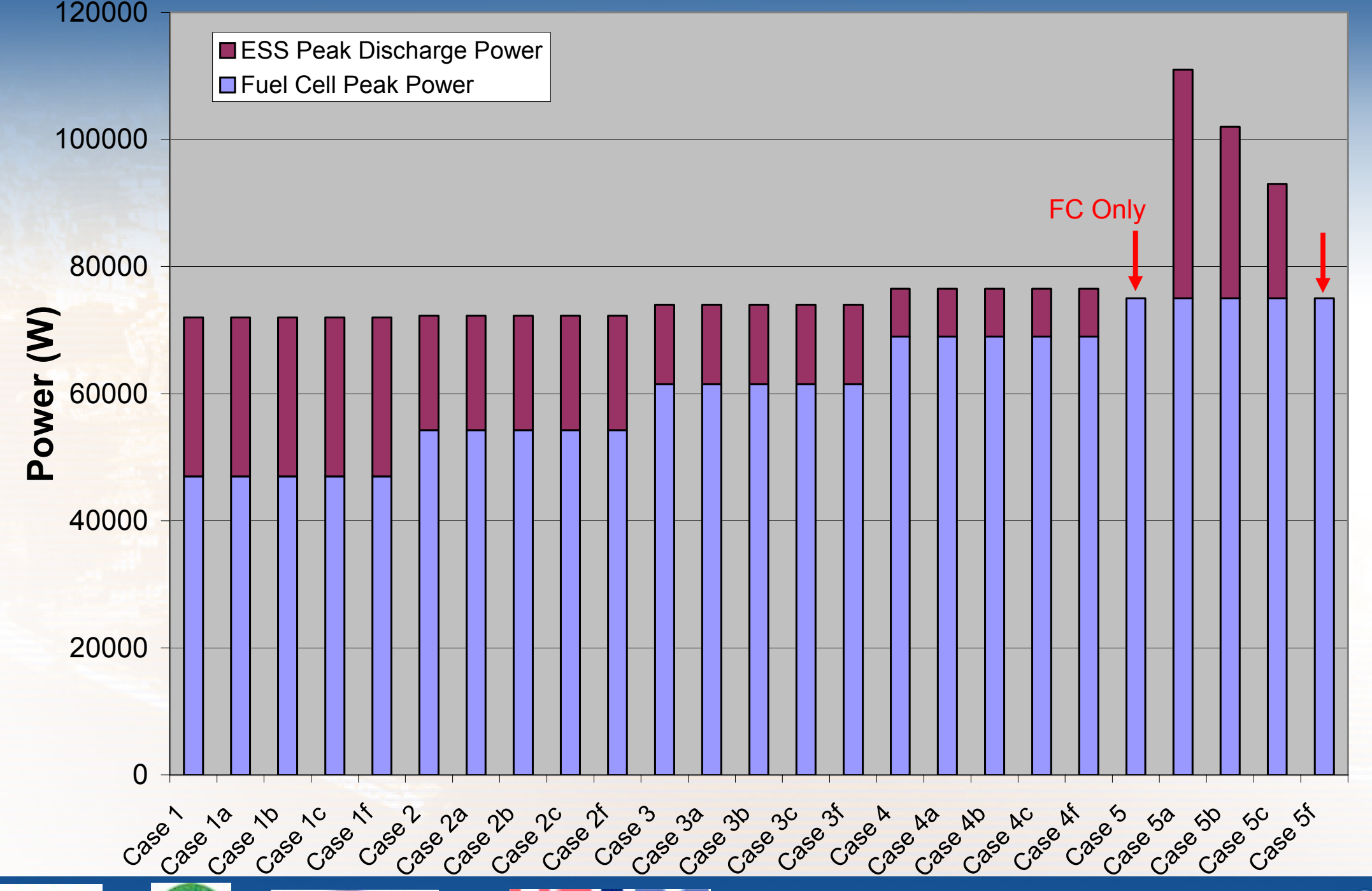




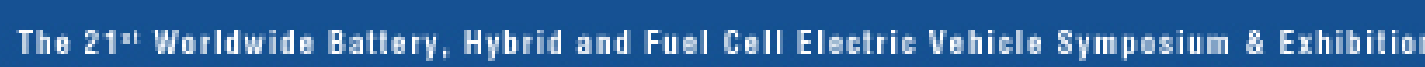

EIS 21 MD ONAGO

2 - 5 April 2005

\section{Preferred Usable
Energy Window for EES \\ Preferred Usable
Energy Window for EES} tatis

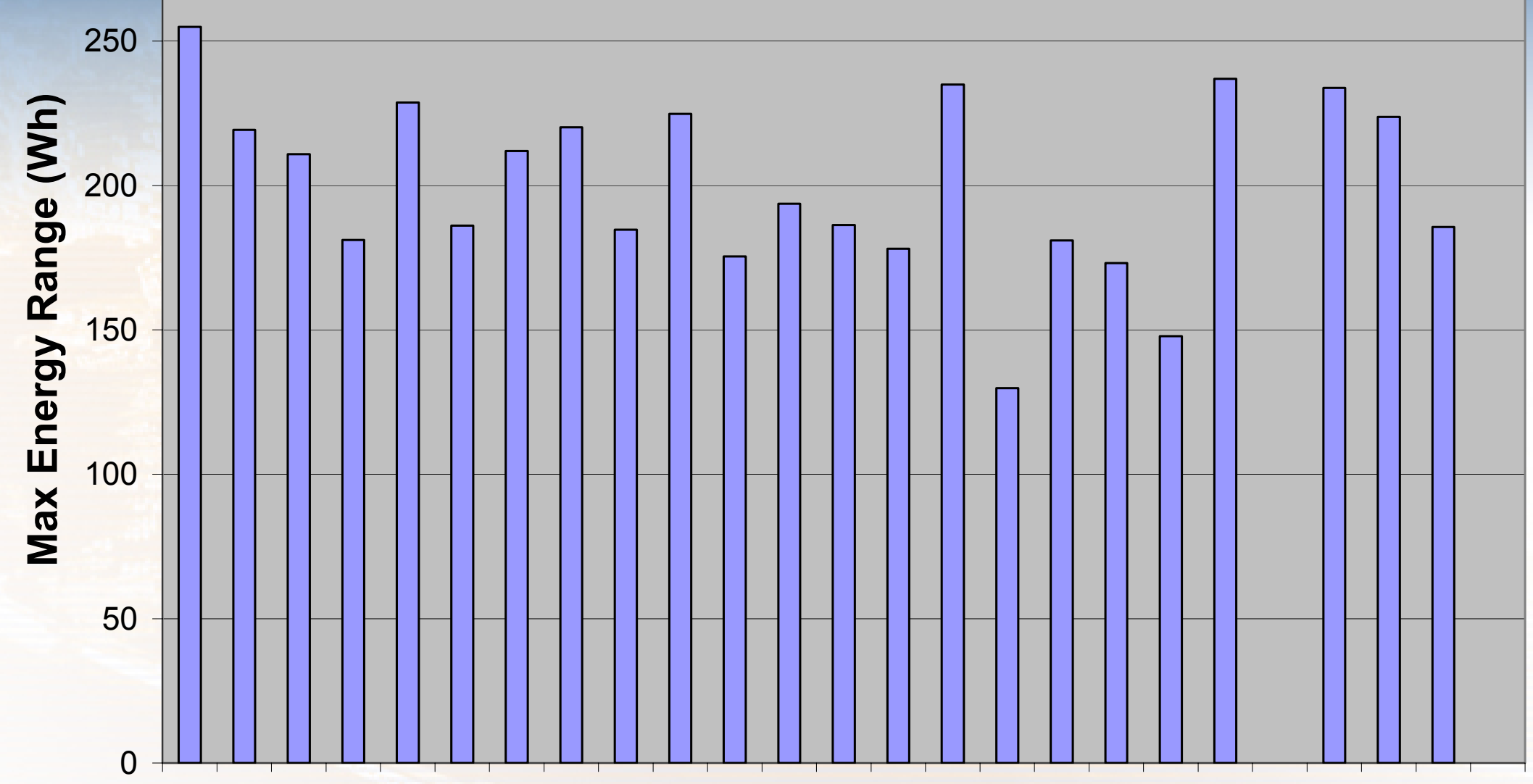

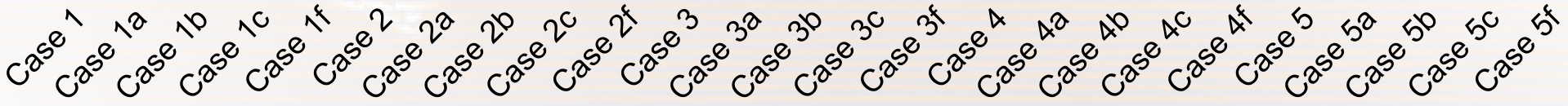

\section{"NREI}




\section{EISS 21 MONACO}

2 - 5 April 2005

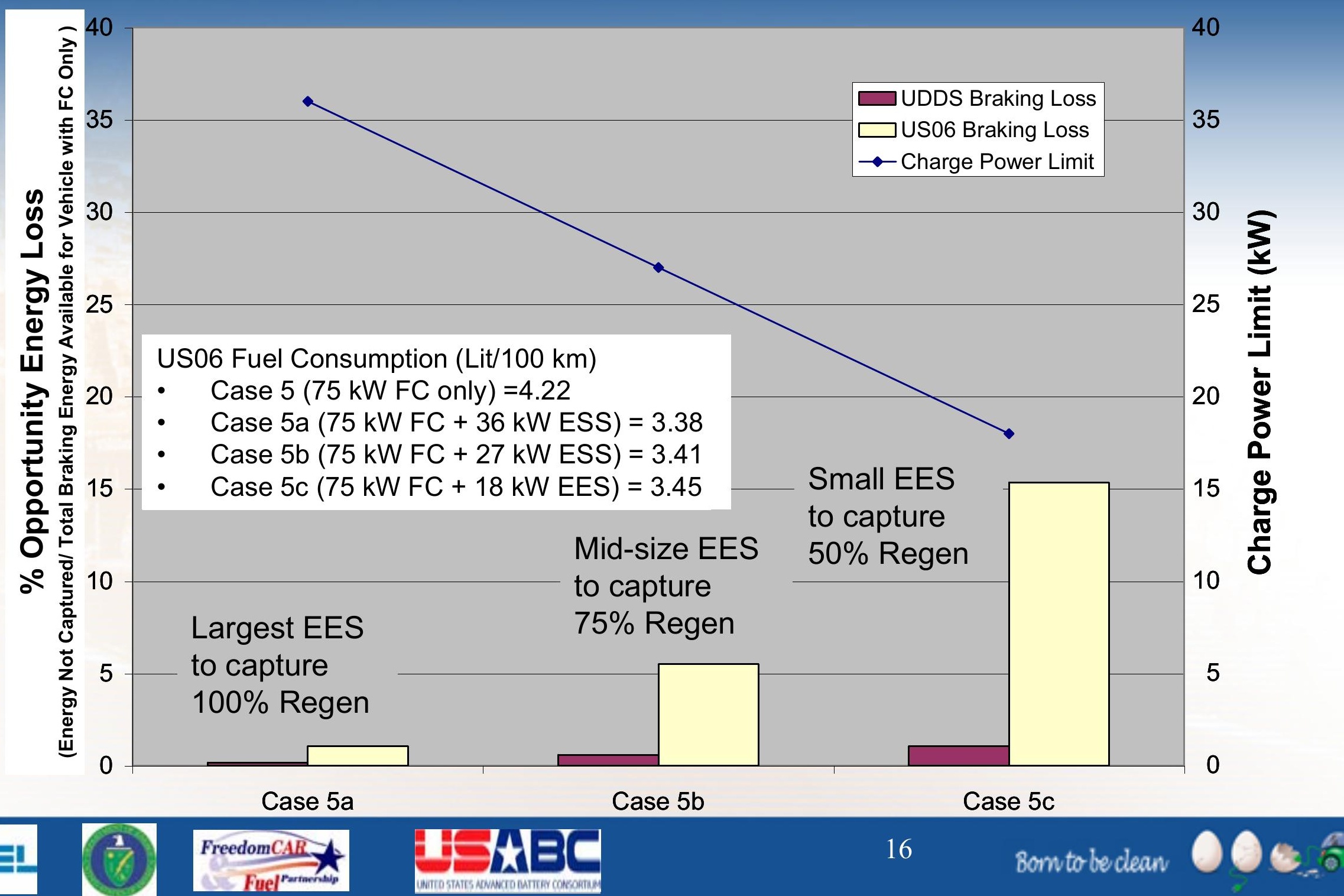


- Comparing Case 5 (FC only) to all others, demonstrates advantage of having any ESS on-board

- Very best fuel consumption for Case $1 \mathrm{f}$

- Smallest fuel cell

- ESS for $50 \%$ regen power

- Fuel cell peak efficiency at $10 \%$ rated power

$3 \mathrm{~L} / 100 \mathrm{~km} \sim 80 \mathrm{MPG} \sim 0.8 \mathrm{~kg} \mathrm{H} / 100 \mathrm{~km}$

2

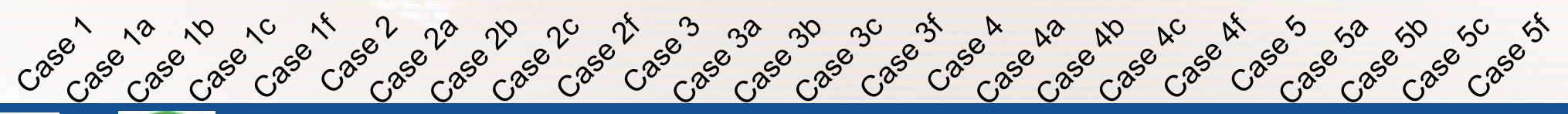




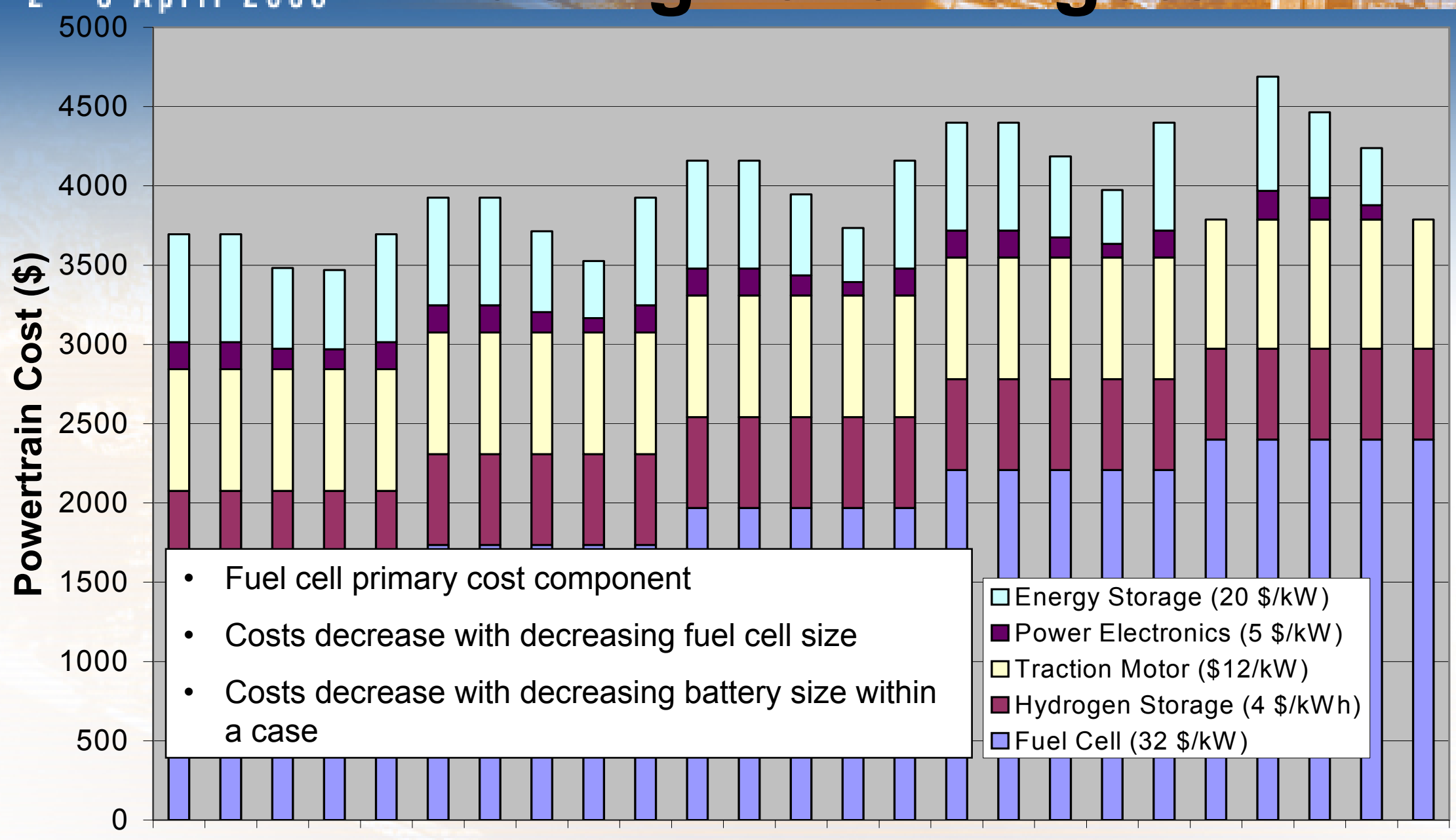

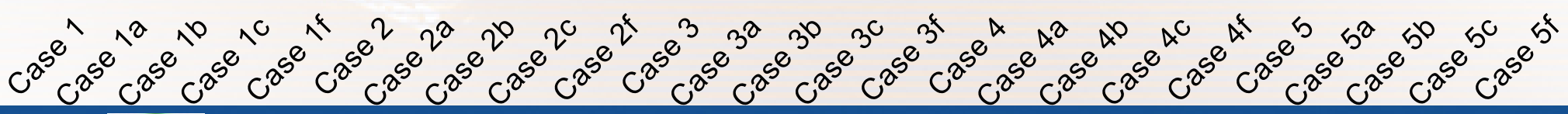


EISS 21 MONACO 2 - 5 April 2005 0.025

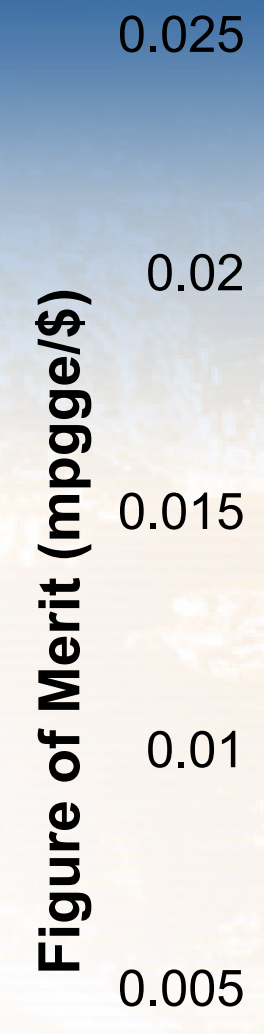

0

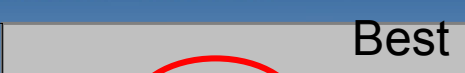
Choosing a Scenario
Figure of Merit (Fuel Economy/Cost) Cases

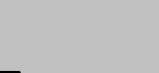
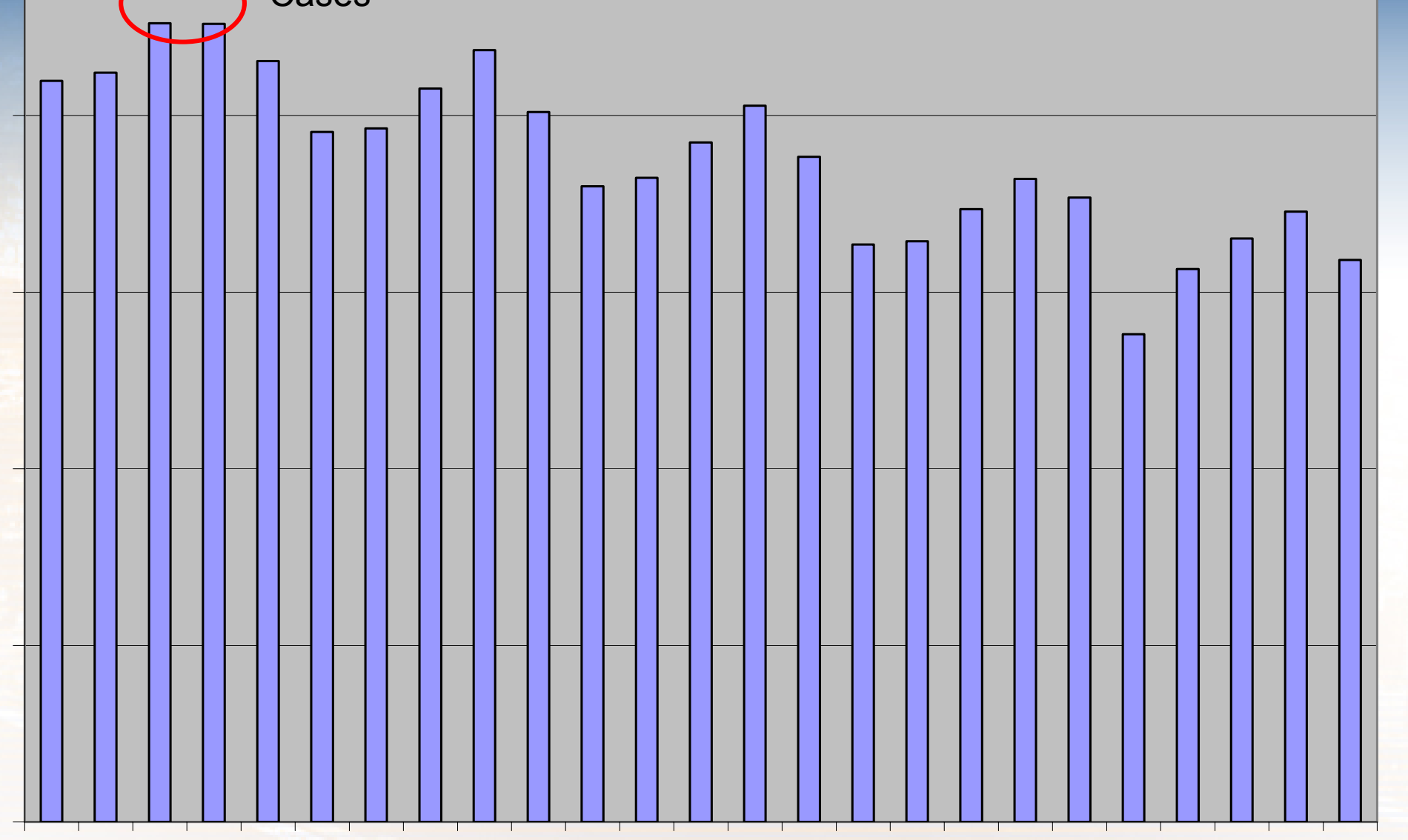

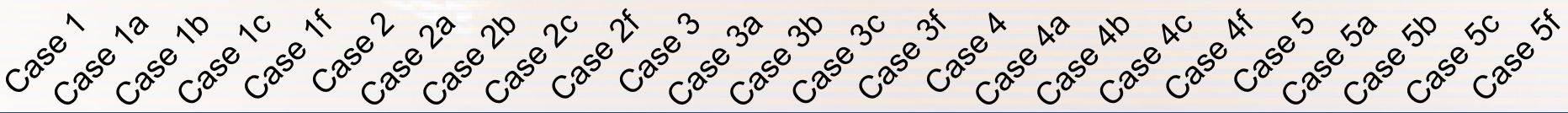

Higher fuel economy, lower cost

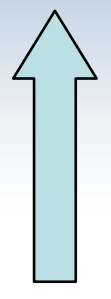<smiles>C1CCC1</smiles>

Lower fuel economy, higher cost

\section{WNPEI}




\section{2 - 5 April 2005}
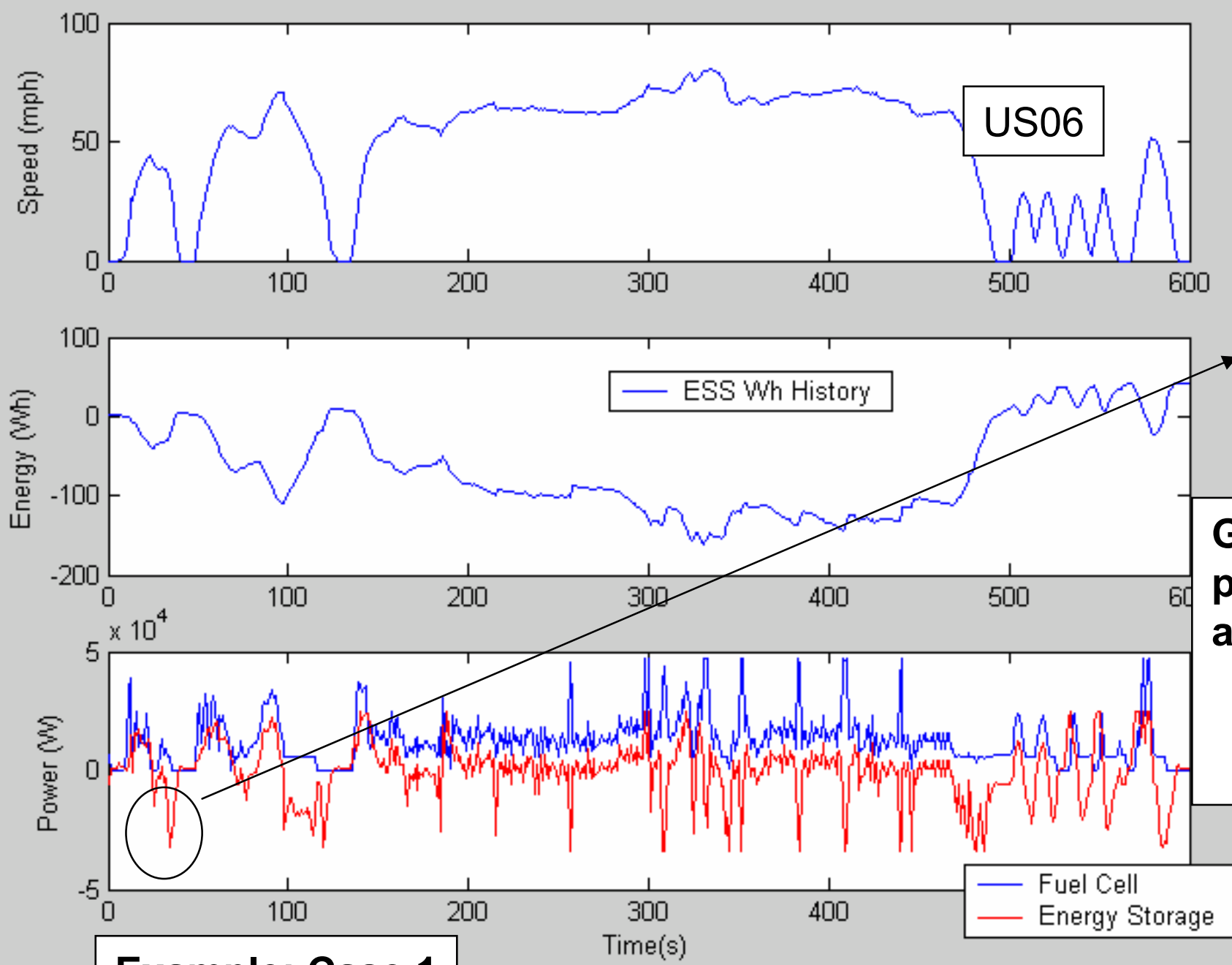

Given typical ESS power profile, what would be the appropriate:

- Discharge power (x s)

- Regen power (y s)

- Energy range (Wh)?

Example: Case 1 
The 21" Worldwide Battery, Hybrid and Fuel Cell Electric Vehicle Symposium \& Exhibition

EIS 21 DO ONACO

2 - 5 April 2005
Analysis of ESS Power Profile

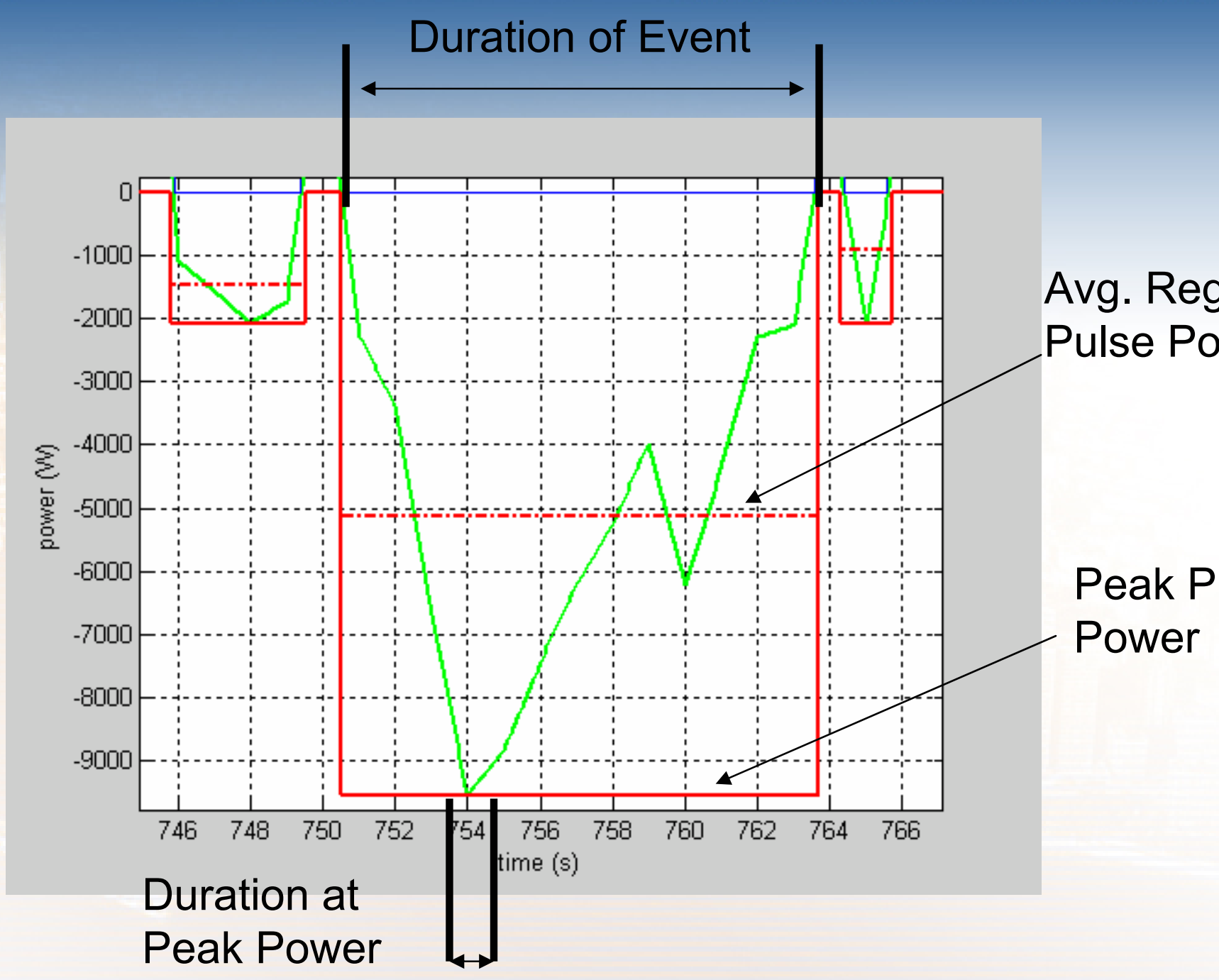




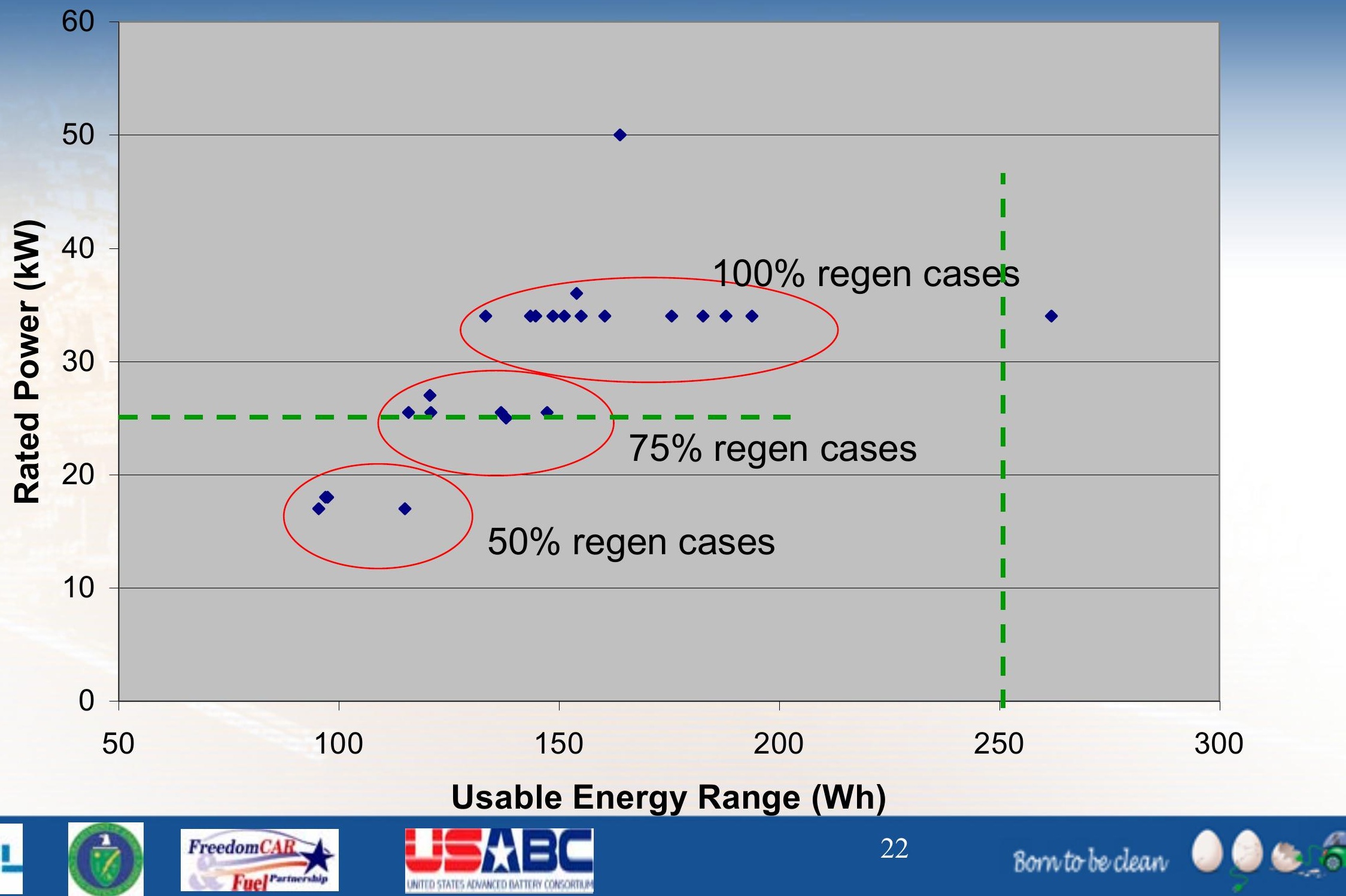


- Peak power events

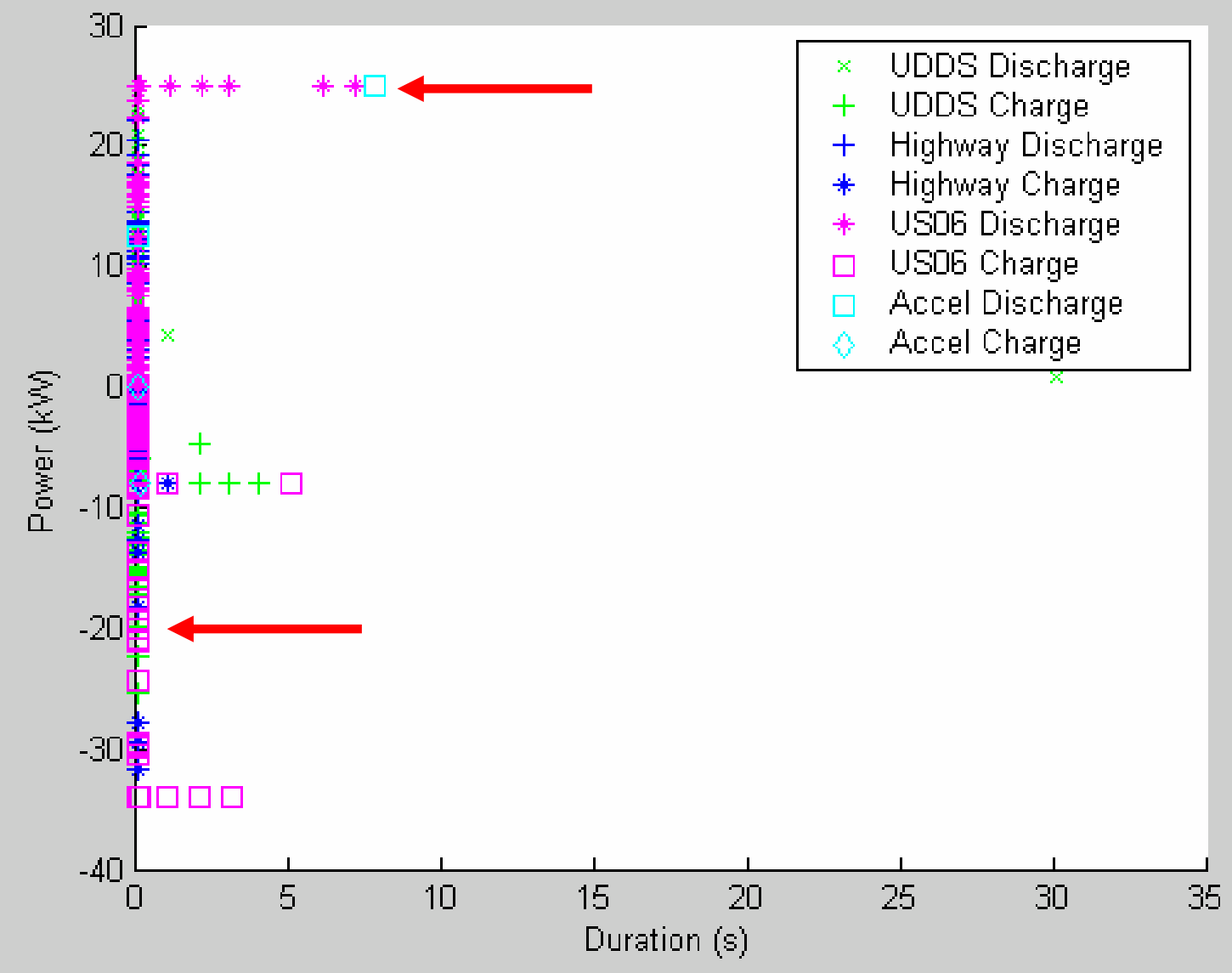
typically only last for short duration (artifact of study approach)

- Discharge sized for acceleration

- Charge sized for US06 
EI/S 21 Avg. Power Need vS. Duration DO ONAGO

2 - 5 April 2005

\section{Data for Multiple Cycles}

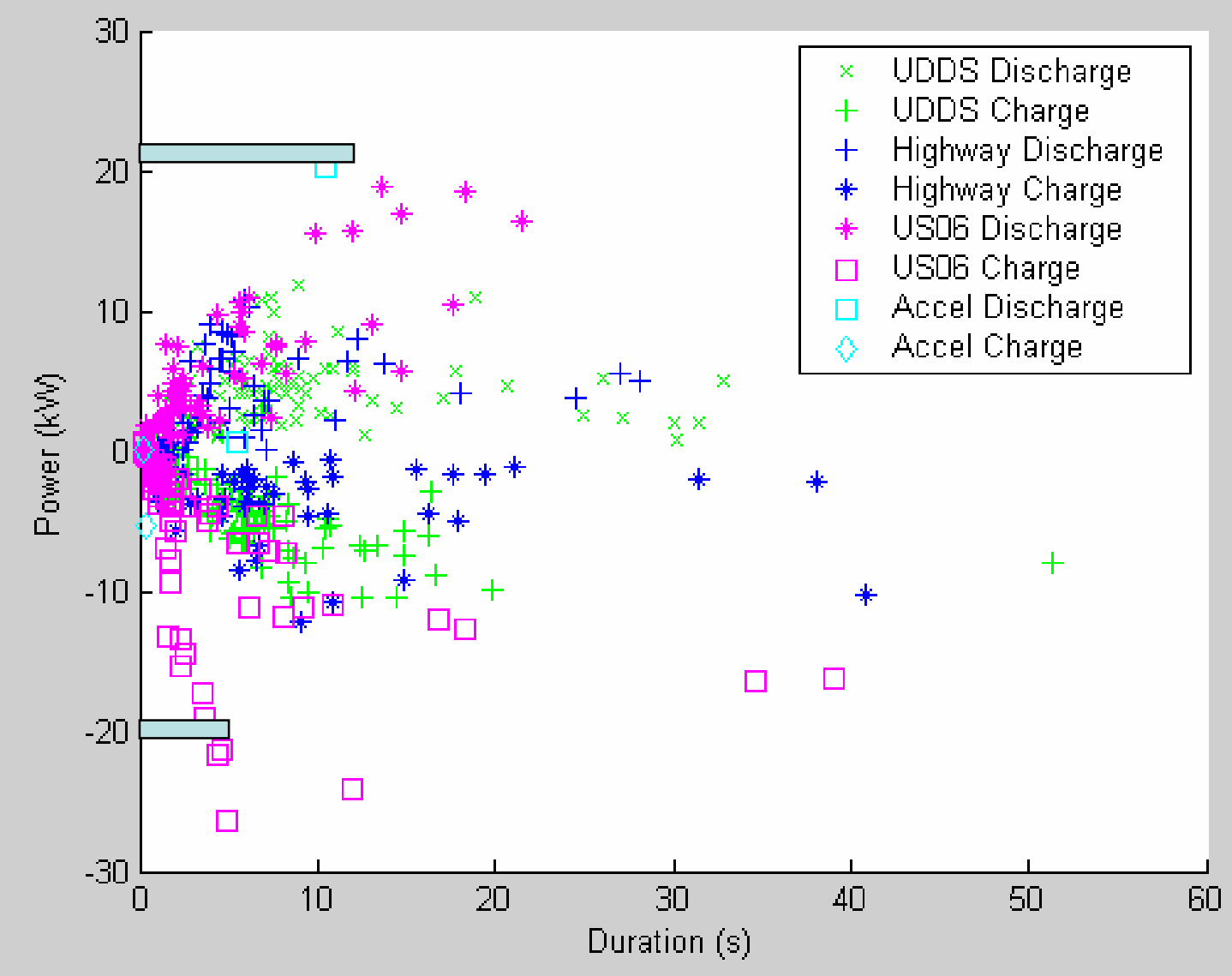

- Power is average power of an event (energy/duration)

- Duration is the time from 0 to 0 power

- Acceleration performance sets discharge requirements

- US06 cycle sets charge requirements 
(Smallest fuel cell with moderate ESS)

(For a light-weight, aerodynamic, mid-size car)

\begin{tabular}{|l|c|c|}
\hline \multicolumn{1}{|c|}{$\begin{array}{c}\text { Goal } \\
\text { (Specifications) }\end{array}$} & (units) & ESS for FCV \\
\hline Pulse Discharge Power (12 s) & $\mathrm{kW}$ & 25 \\
\hline Max Regen Pulse (5 s) & $\mathrm{kW}$ & 20 \\
\hline Available Energy & $\mathrm{Wh}$ & 250 \\
\hline
\end{tabular}




\section{Conclusions}

2 - 5 April 2005

- Intelligent energy management strategy to capture and utilize regen energy in fuel cell hybrid vehicle is critical

- In general, $25-30 \%$ improvement in fuel consumption from hybridization

- As long as regen capture is maximized - regen strategy not critical

- smallest fuel cell with moderate ESS was the most fuel efficient, but also the least expensive scenario

- ESS with 200-250 Wh of usable range seems sufficient for assumed lightweight midsize fuel cell car

- ESS with regen power of around $20 \mathrm{~kW}$ for $5 \mathrm{~s}$ and discharge power of $25 \mathrm{~kW}$ for 10-15s appears to be sufficient for lightweight midsize fuel cell car

- Planning to perform sensitivity analysis and investigate other opportunities provided by fuel cell operating strategies 